\title{
DIM and diagnostic placement for NIF experiments
}

\author{
Dan Kalantar
}

September 14, 1999

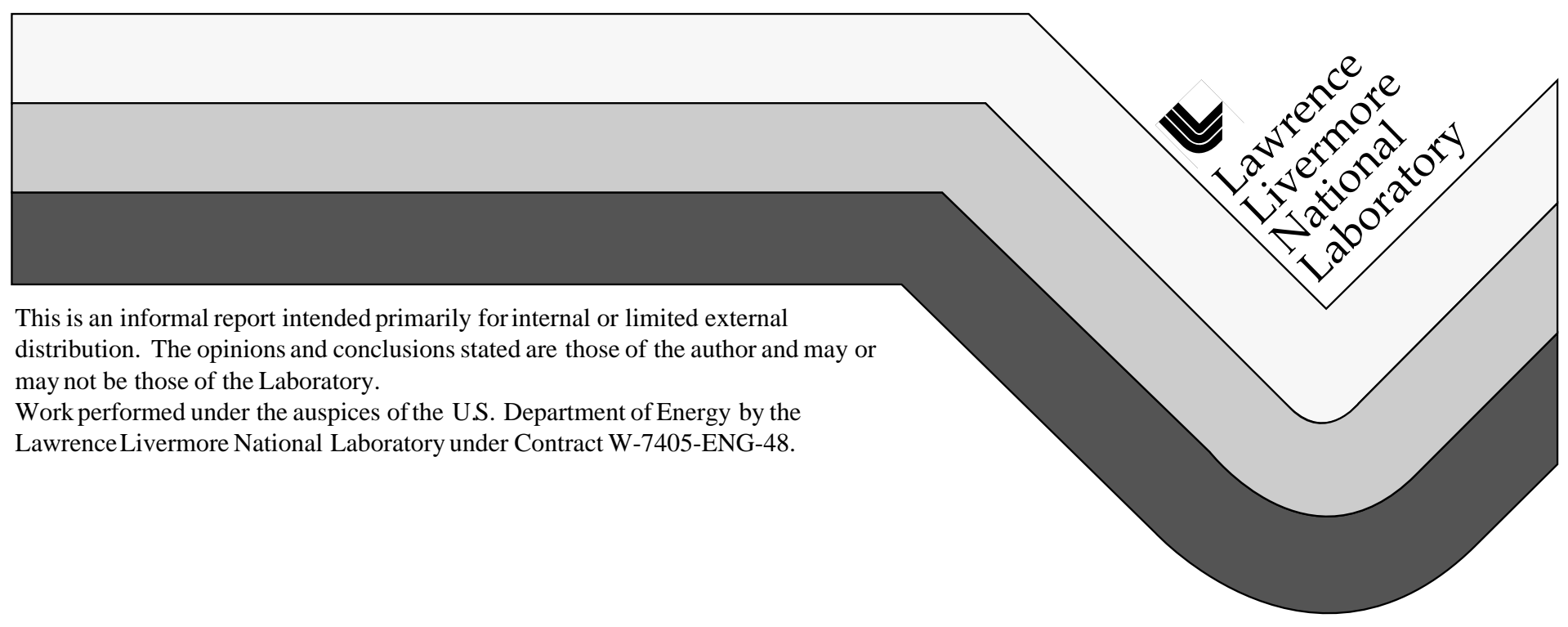




\section{DISCLAIMER}

This document was prepared as an account of work sponsored by an agency of the United States Government. Neither the United States Government nor the University of California nor any of their employees, makes any warranty, express or implied, or assumes any legal liability or responsibility for the accuracy, completeness, or usefulness of any information, apparatus, product, or process disclosed, or represents that its use would not infringe privately owned rights. Reference herein to any specific commercial product, process or service by trade name, trademark, manufacturer, or otherwise, does not necessarily constitute or imply its endorsement, recommendation, or favoring by the United States Government or the University of California. The views and opinions of authors expressed herein do not necessarily state or reflect those of the United States Government or the University of California, and shall not be used for advertising or product endorsement purposes.

This report has been reproduced Directly from the best available copy.

Available to DOE and DOE contractors from the Office of Scientific and Technical Information

P.O. Box 62, Oak Ridge, TN 37831

Prices available from (615) 576-8401, FTS 626-8401

Available to the public from the National Technical Information Service

U.S. Department of Commerce 5285 Port Royal Rd., Springfield, VA 22161 
Mail Station L-473

Ext: 2-6147

September 14, 1999

NIF-0035359

WBS 1.8.3.2

To: $\quad$ Distribution

From: Dan Kalantar

Subject: DIM and diagnostic placement for NIF experiments

The input that has been provided on the NIF experiment setup sheets has allowed us to review the diagnostic and DIM placement as well as the baseline unconverted light management plan. We have done an iteration to identify common diagnostic lines of sight, and with additional requirements defined by specific experiments, we propose (1) a baseline plan for DIM placement requiring only five DIMs that may be moved between up to seven DIM ports, and (2) a modified baseline unconverted light management plan. We request additional input to identify primary $v$ s. secondary diagnostics for each experiment definition. Feedback is requested by October 15.

\section{Baseline DIM placement}

\begin{tabular}{|l|l|l|}
\hline NIF port & Diagnostic & Comments \\
\hline $0-0$ & DIM & Top view, face-on for vert'l halfraum, axial for hohlraum \\
\hline $90-213$ & DIM & Waist view, orthogonal to 90-123 \\
\hline $90-315$ & DIM & Waist view, side-on, orthogonal to 90-45 \\
\hline $90-348$ & DIM & Waist view, preferred for symmetry expts \\
\hline $90-45$ & DIM & Waist view, side-on, orthogonal to $90-315$ \\
\hline $90-123$ & DIM & Waist view, aligned with inner cone beams in hohlraum \\
\hline $116-129$ & DIM & Below waist, near to Cluster 4 beams \\
\hline $18-123$ & SXI & Within $20^{\circ}$ of vertical axis, near top \\
\hline $161-236$ & SXI & Within $20^{\circ}$ of vertical axis, near bottom \\
\hline $90-174$ & Drive & LEH view for horiz'l halfraum, waist view for hohlraum \\
\hline $143-94$ & Drive & View in LEH for vertical hohlraums \\
\hline
\end{tabular}

NOTE: DIM must be moved from nearby location to be used on ports shown as shaded.

The NIF will be outfitted with a number of DIMs to allow flexibility in experiment design. We have used the input from users to identify common 
diagnostic placements. This leads to a baseline port assignment that will be used during construction in locating the utilities required for initial DIM placement as listed in the table, above.

We show a breakdown of the proposed DIM locations in Table 1 as a function of phase. The priority and timeline are discussed below.

\section{Activation}

For first light in the target chamber, we require three DIMs with views on the waist for beam synchronization tests, and above and below the waist for $\mathrm{x}$-ray spot imaging. Additionally, we require the static x-ray imagers (SXIs) on ports near the top and bottom of the chamber for beam pointing verification. The port assignments to accommodate these requirements are listed in Table 1a.

\section{Cluster 4 operation}

For Cluster 4 operation, we show two additional DIM locations at 90-315 and 9045 in Table 1b. The DIM location at 90-315 is required for face-on imaging of horizontal halfraum experiments. The orthogonal line of sight at $90-45$ is required for side-on imaging of horizontal halfraum experiments. However, this line of sight is duplicated by the top DIM view (0-0) for many experiments. It is likely that we will be able to move another nearby DIM to this location when this line of sight is required. As a result, this DIM location is shown shaded, and a minimum of four DIMs will be required. In addition to the DIM ports, we require a soft x-ray power diagnostic with a view into a horizontal hohlraum. This is accomplished with the line of sight at 90-174. This line of sight is compatible with a DIM, which means the power diagnostic may be DIM based. In this case, an additional DIM will be required for this location.

\section{Symmetric half NIF}

For 96-beam symmetric operation, there will start to be vertical hohlraum experiments. In order to maintain simultaneous face-on and side-on lines of sight for physics packages mounted off the midplane of the hohlraum, we require both the 90-45 and 90-315 DIM views. Combining this requirement with DIM views above (top) and below the midplane and a near-opposite view (90213), we require a total of 5 DIMs (Table 1c).

For x-ray power measurements on vertical hohlraum and halfraum targets, we require the capability to view the target in the midplane and also through the LEH. The midplane view is provided by the port at 90-174. We identify the additional line of sight through the LEH at 143-94. This view is not compatible with a DIM due to an interference with the target bay floor, so this power diagnostic must be hard mounted.

\section{Full NIF}

In order to accommodate requirements for imaging along a line of sight that intersects a minimum number of inner cone beam spots in the hohlraum, we include the orthogonal lines of sight at 90-123 (already assigned for Cluster 4 operation) and 90-213 (new). An additional line of sight at 90-348 is included to allow multiple diagnostic views from the same side. Both of these additional 
DIM lines of sight are shown with shading in Table 1d. The experiments that require these additional lines of sight may be accommodated by moving DIMs from nearby port locations.

The diagnostic and DIM port assignments are shown on a map of the target chamber in Figure 1. This figure also shows lines of sight in $\Theta, \varnothing$ that are impacted by focused dispersed $1 \mathrm{w}$ laser light, as described below. The proposed lines of sight identified for DIM placement are not affected by the dispersed unconverted light coming to best focus.

\section{Unconverted light management plan}

In order to accommodate the diagnostic line of sight requirements for Cluster 4 and later operation, we propose a modified distribution for the unconverted light by re-assigning the locations for some of the color separation gratings (CSGs) on NIF (see document NIF-5001588). In Figure 2 we show (a) the distribution of unconverted light near target chamber center as illustrated in NIF document 5001588 and (b) the proposed distribution of unconverted light. These images represents the intersection of the dispersed unconverted light in the horizontal midplane of the target chamber (not to scale). In this plane, the light is generally confined in two $45^{\circ}$ segments. The proposed changes effectively rotate the two wedges in ø. Note that these two segments are clear of all diagnostic lines of sight identified in Table 1.

Away from chamber center, the 1w light comes to best focus about $46 \mathrm{~cm}$ beyond the $3 \mathrm{w}$ focus. Since the dispersed orders may focus to a high intensity, we need to restrict the diagnostics from intersecting a point of best focus for this unconverted light. The $\Theta, \varnothing$ locations corresponding to best focus for one of the first 20 diffracted orders are marked by the many square points overlaid on the chamber port map in Figure 1. The diagnostic port assignments that we identify above are compatible with the distribution of high intensity light.

\section{Feedback}

The diagnostic lines of sight and proposed DIM port assignments have been developed based on input in the form of NIF experiment configurations. We have modified experiment layouts to make them more compatible with common lines of sight. However, further detail is required to identify primary vs. secondary diagnostics and to verify that the experiments may fit within the proposed port assignments. Any feedback that may impact the port assignments must be provided by October 1 . 
Table 1: Proposed diagnostic port assignments

\section{a) First light}

\begin{tabular}{|l|l|l|}
\hline NIF port & Diagnostic & Comments \\
\hline $0-0$ & DIM & Top view \\
\hline $90-123$ & DIM & Waist view, within Cluster 4 beams \\
\hline $116-129$ & DIM & Below waist, near to Cluster 4 beams \\
\hline $18-123$ & SXI & Within $20^{\circ}$ of vertical axis, near top \\
\hline $161-236$ & SXI & Within $20^{\circ}$ of vertical axis, near bottom \\
\hline
\end{tabular}

\section{b) Cluster 4 operation}

\begin{tabular}{|l|l|l|}
\hline NIF port & Diagnostic & Comments \\
\hline $0-0$ & DIM & Top view, side-on for horizontal hohlraum \\
\hline $90-315$ & DIM & Waist view, axial for horizontal halfraum \\
\hline $90-45$ & DIM & Waist view, side-on for horizontal halfraum \\
\hline $90-123$ & DIM & LEH view for horizontal halfraum \\
\hline $116-129$ & DIM & Below waist, near to Cluster 4 beams, LEH \\
\hline $18-123$ & SXI & Within $20^{\circ}$ of vertical axis, near top \\
\hline $161-236$ & SXI & Within $20^{\circ}$ of vertical axis, near bottom \\
\hline $90-174$ & Drive & LEH view for horiz'l halfraum, waist view for hohlraum \\
\hline
\end{tabular}

NOTE: DIM must be moved from nearby location to be used on ports shown as shaded.

c) Symmetric half NIF

\begin{tabular}{|l|l|l|}
\hline NIF port & Diagnostic & Comments \\
\hline $0-0$ & DIM & Top view, face-on for vert'l halfraum, axial for hohlraum \\
\hline $90-315$ & DIM & Waist view, side-on, orthogonal to 90-45 \\
\hline $90-45$ & DIM & Waist view, side-on, orthogonal to $90-315$ \\
\hline $90-123$ & DIM & Waist view, aligned with inner cone beams in hohlraum \\
\hline $116-129$ & DIM & Below waist, near to Cluster 4 beams \\
\hline $18-123$ & SXI & Within $20^{\circ}$ of vertical axis, near top \\
\hline $161-236$ & SXI & Within $20^{\circ}$ of vertical axis, near bottom \\
\hline $90-174$ & Drive & LEH view for horiz'l halfraum, waist view for hohlraum \\
\hline $143-94$ & Drive & View in LEH for vertical hohlraums \\
\hline
\end{tabular}


Table 1 (continued)

d) Full NIF

\begin{tabular}{|l|l|l|}
\hline NIF port & Diagnostic & Comments \\
\hline $0-0$ & DIM & Top view, face-on for vert'1 halfraum, axial for hohlraum \\
\hline $90-213$ & DIM & Waist view, orthogonal to 90-123 \\
\hline $90-315$ & DIM & Waist view, side-on, orthogonal to 90-45 \\
\hline $90-348$ & DIM & Waist view, preferred for symmetry expts \\
\hline $90-45$ & DIM & Waist view, side-on, orthogonal to 90-315 \\
\hline $90-123$ & DIM & Waist view, aligned with inner cone beams in hohlraum \\
\hline $116-129$ & DIM & Below waist, near to Cluster 4 beams \\
\hline $18-123$ & SXI & Within $20^{\circ}$ of vertical axis, near top \\
\hline $161-236$ & SXI & Within $20^{\circ}$ of vertical axis, near bottom \\
\hline $90-174$ & Drive & LEH view for horiz'l halfraum, waist view for hohlraum \\
\hline $143-94$ & Drive & View in LEH for vertical hohlraums \\
\hline
\end{tabular}

NOTE: DIM must be moved from nearby location to be used on ports shown as shaded. 
Figure 1: Chamber map with diagnostic and DIM assignments labeled. The $\Theta, \varnothing$ points for lines of sight impacted by best focus for dispersed orders of unconverted light are shown as small squares overlaid on the map.

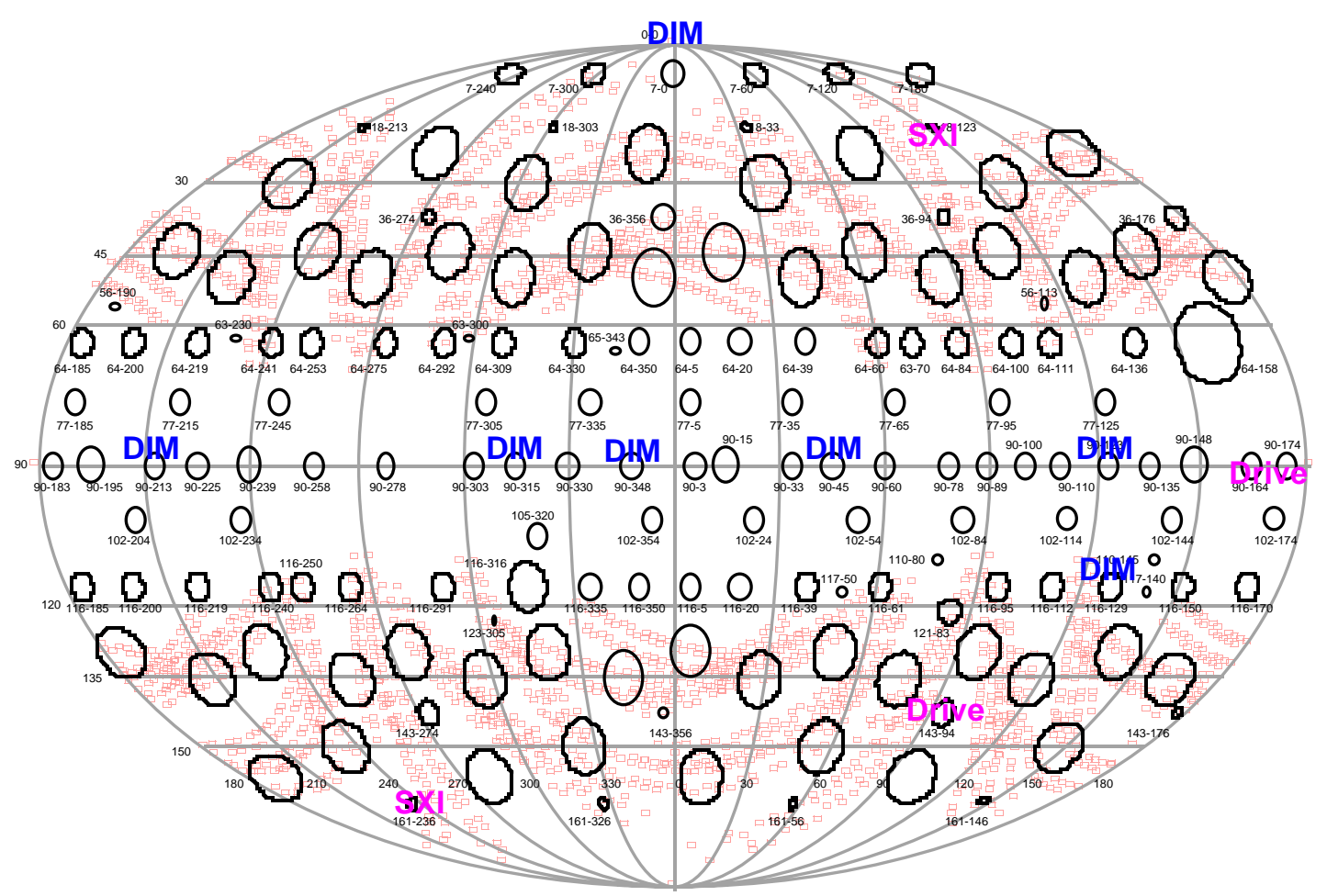


Figure 2: (a) Distribution of unconverted light from Baseline Unconverted Light Management Plan (NIF-5001588). (b) Modified distribution of unconverted light achieved by re-assigning CSGs. The distribution of unconverted light is shown only in a reqion $10 \mathrm{~cm}$ square near target chamber center.

a)

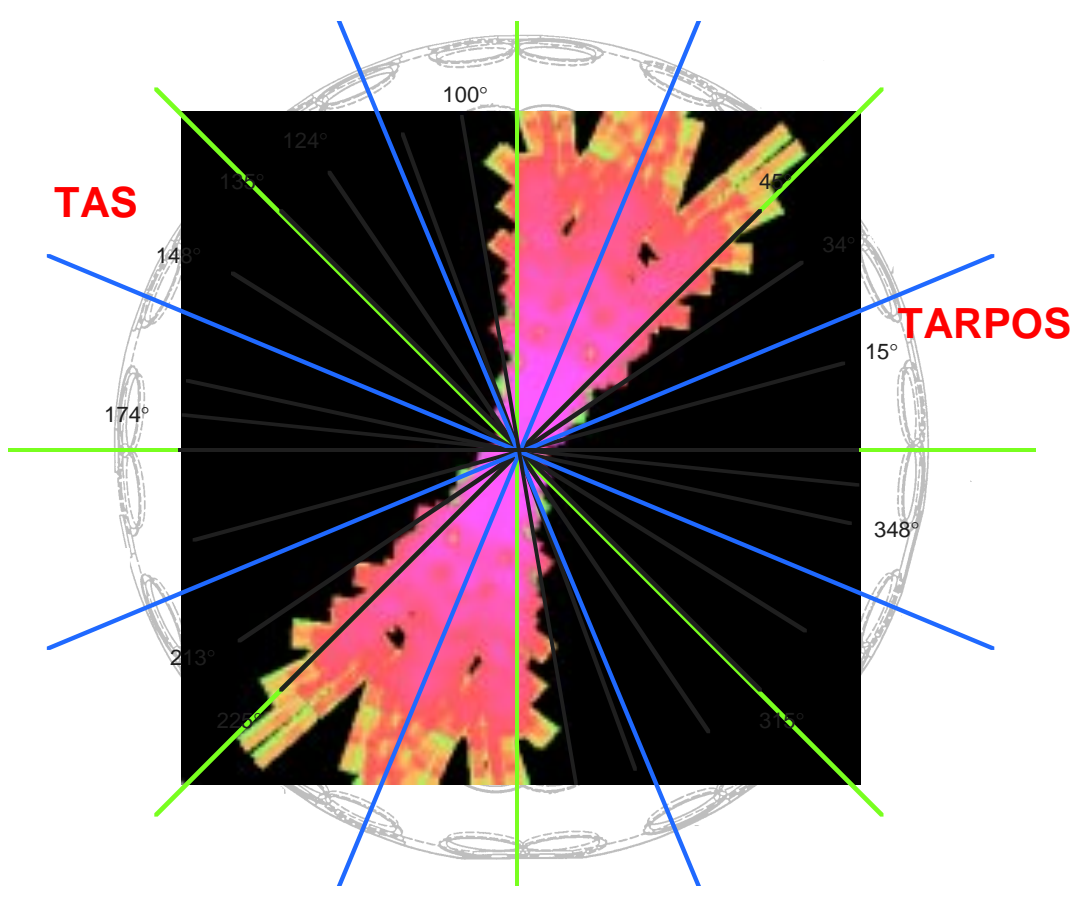

b)

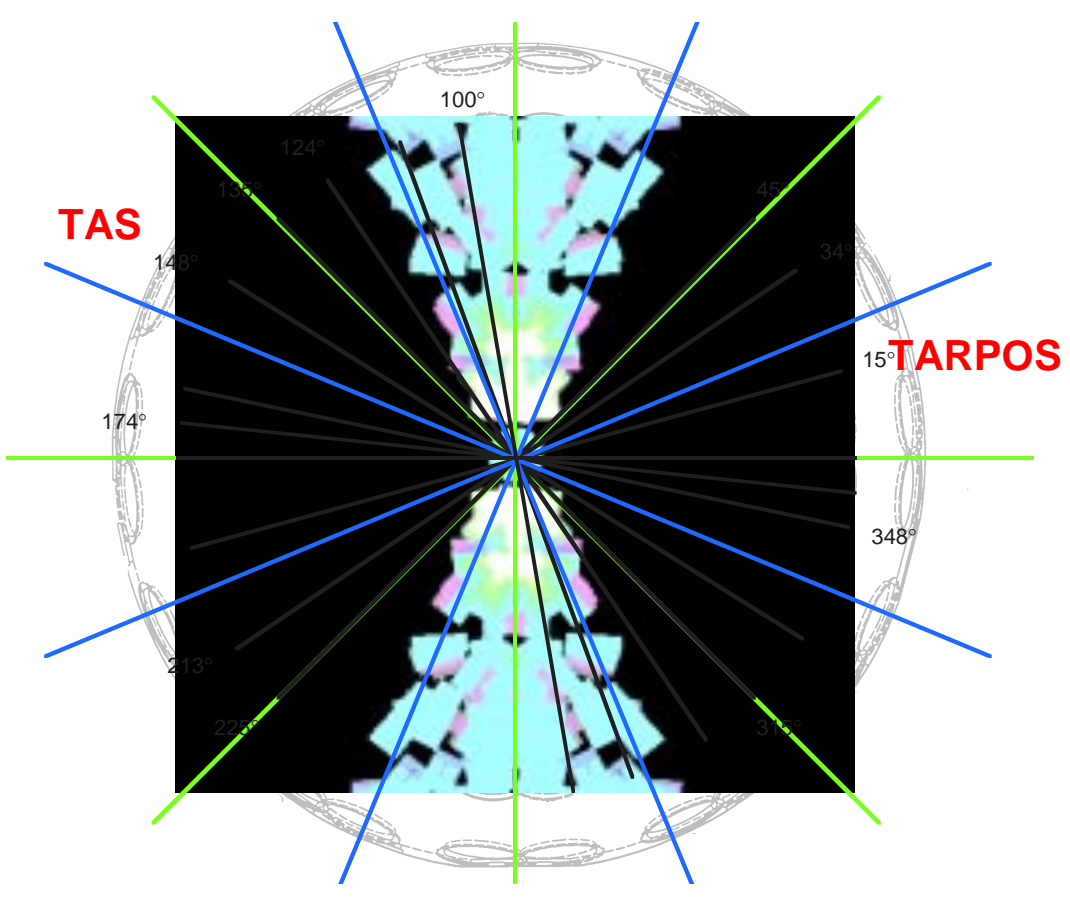

